\title{
Enhanced salt sensitivity following shRNA silencing of neuronal TRPV1 in rat spinal cord
}

\author{
Shuang-quan $\mathrm{YU}^{1}$, Donna H WANG ${ }^{1,2, *}$ \\ ${ }^{1}$ Department of Medicine, Michigan State University, East Lansing, Michigan, USA; ${ }^{2}$ Neuroscience Program and Cell and Molecular \\ Biology Program, Michigan State University, East Lansing, Michigan, USA
}

\begin{abstract}
Aim: To investigate the effects of selective knockdown of TRPV1 channels in the lower thoracic and upper lumbar segments of spinal cord, dorsal root ganglia (DRG) and mesenteric arteries on rat blood pressure responses to high salt intake.

Methods: TRPV1 short-hairpin RNA (shRNA) was delivered using intrathecal injection ( $6 \mu \mathrm{g} \cdot \mathrm{kg}^{-1} \cdot \mathrm{d}^{-1}$, for $3 \mathrm{~d}$ ). Levels of TRPV1 and tyrosine hydroxylase expression were determined by Western blot analysis. Systolic blood pressure and mean arterial pressure (MAP) were examined using tail-cuff and direct arterial measurement, respectively.

Results: In rats injected with control shRNA, high-salt diet (HS) caused higher systolic blood pressure compared with normal-salt diet (NS) (HS:149 $\pm 4 \mathrm{mmHg}$; NS:126 $\pm 2 \mathrm{mmHg}, P<0.05)$. Intrathecal injection of TRPV1 shRNA significantly increased the systolic blood pressure in both HS rats and NS rats (HS:169 $\pm 3 \mathrm{mmHg}$; NS:139 $\pm 2 \mathrm{mmHg}$ ). The increases was greater in HS rats than in NS rats (HS: 13.9\% $\pm 1.8 \%$; NS: 9.8 $\pm 0.7, P<0.05)$. After TRPV1 shRNA treatment, TRPV1 expression in the dorsal horn and DRG of T8-L3 segments and in mesenteric arteries was knocked down to a greater extent in HS rats compared with NS rats. Blockade of $\alpha 1$-adrenoceptors abolished the TRPV1 shRNA-induced pressor effects. In rats injected with TRPV1 shRNA, level of tyrosine hydroxylase in mesenteric arteries was increased to a greater extent in HS rats compared with NS rats.

Conclusion: Selective knockdown of TRPV1 expression in the lower thoracic and upper lumbar segments of spinal cord, DRG, and mesenteric arteries enhanced the prohypertensive effects of high salt intake, suggesting that TRPV1 channels in these sites protect against increased salt sensitivity, possibly via suppression of sympatho-excitatory responses.
\end{abstract}

Keywords: blood pressure; high salt intake; intrathecal injection; short-hairpin RNA; sympathetic nerve; TRPV1

Acta Pharmacologica Sinica (2011) 32: 845-852; doi: 10.1038/aps.2011.43

\section{Introduction}

Accumulating evidence indicates that sensory nerves play a key role in regulating blood pressure $\mathrm{e}^{[1-3]}$. The transient receptor potential vanilloid type 1 (TRPV1) channel is abundantly expressed in sensory nerves ${ }^{[4,5]}$ and conveys the intricate task of modulating the function of these nerves. Thus, defining the role of TRPV1-positive sensory nerves in the regulation of blood pressure and salt sensitivity will be useful. We have shown previously that the degeneration of TRPV1-expressing sensory nerves throughout the neonatal body by subcutaneous injection of capsaicin, a selective TRPV1 agonist, leads to increased salt sensitivity of arterial pressure, indicating that TRPV1-positive sensory nerves play a counter-regulatory role against salt-induced increases in blood pressure ${ }^{[6-20]}$. The underlying mechanism of anti-hypertensive effects of TRPV1

\footnotetext{
* To whom correspondence should be addressed.

E-mail donna.wang@ht.msu.edu

Received 2011-02-22 Accepted 2011-04-07
}

may involve its counter-balancing role against the activation of the renin-angiotensin-aldosterone system ${ }^{[7-9]}$, sympathetic nervous system ${ }^{[10]}$, endothelin system ${ }^{[11,12]}$, superoxide generation system ${ }^{[13,14]}$, and epithelial sodium transporters ${ }^{[15]}$. Our previous approach using systemic sensory denervation does, however, have two major limitations. First, systemic sensory denervation obliterates not only TRPV1 but also other components that colocalize with TRPV1 in the same neuron, which may regulate blood pressure independently of TRPV1 ${ }^{[21,22]}$. Second, systemic sensory denervation prevents elucidation of the role of specific organ(s) or tissue(s) in regulating blood pressure.

To bypass these limitations, we developed a new model with improved approaches and validation. We applied TRPV1 shRNA to selectively knock down TRPV1 expression without affecting other components that colocalize with TRPV1 in the same neuron. The strategy of using shRNA to selectively silence TRPV channels in vivo has been successfully used in our previous study to effectively knock down the 
expression and function of TRPV4 channels ${ }^{[23]}$. In the present study, we used an intrathecal injection technique to target the T8-L3 segments of the dorsal root ganglia (DRG) and their central (dorsal horn) and peripheral (mesenteric arteries and kidneys) terminals without affecting other regions. The T8-L3 segments were chosen as targets because they innervate mesenteric resistance arteries and the kidneys, which are known for their involvement in blood pressure regulation ${ }^{[24-29]}$.

Using this strategy, we sought to determine whether selective knockdown of TRPV1 expressed in DRG of lower thoracic and upper lumbar segments (T8-L3) and their central and periphery projections enhances the prohypertensive effects induced by salt loading. Furthermore, to assess whether the sympathetic nervous system is involved in TRPV1 shRNAinduced blood pressure regulation, the content of tyrosine hydroxylase (TH), a marker of sympathetic nerves, and blood pressure responses to prazosin, a blocker of a1-adrenoceptors, were examined.

\section{Materials and methods \\ Animals}

Male Wistar rats (201-225 g, Charles River Laboratory, Wilmington, Massachusetts, USA) were housed in a standard facility. Rats received either a normal-salt (NS) diet $(1 \% \mathrm{NaCl}$ by weight, Harlan Teklad, Madison, Wisconsin, USA) or a highsalt (HS) diet ( $8 \% \mathrm{NaCl}$ by weight, Harlan Teklad, Madison, Wisconsin, USA). One week after dietary treatment, rats underwent surgery for intrathecal catheter implantation and removal of the right kidney. One week after surgery, rats began receiving intrathecal injections of either TRPV1 shRNA or control shRNA once per day for $3 \mathrm{~d}$ and were randomly divided into 4 groups: NS diet+control shRNA, NS diet+TRPV1 shRNA, HS diet+control shRNA, or HS diet+TRPV1 shRNA. Measurement of systolic blood pressure, mean arterial pressure, water intake, and urine excretion and tissue sample collection for immunohistochemistry and Western blot analysis were performed on $\mathrm{d} 3$ after the beginning of shRNA treatment. All experiments were performed in accordance with the protocols approved by the Institutional Animal Care and Use Committee of Michigan State University.

\section{Surgery for intrathecal catheter implantation and unine- phrectomy}

Under anesthetization with ketamine/xylazine $(85 / 5 \mathrm{mg} / \mathrm{kg}$, ip), the nape skin was cut open and a small incision was made in the atlanto-occipital membrane. The muscles were separated, and a PE-10 catheter was inserted into the subarachnoid space as far as the T8 segment of the spinal cord (the length of the catheter from the inside end to the atlanto-occipital membrane was about $7.5 \mathrm{~cm}$ ) and sutured in place, and the incision was closed. About $5 \mathrm{~cm}$ of the catheter remained external for injection of shRNA and was sealed with parafilm to stop the outflow of cerebrospinal fluid when not used for injection. Uninephrectomy was performed by making a right flank incision and then removing the right kidney.
Preparation of TRPV1 shRNA or control shRNA and intrathecal injection

TRPV1 shRNA or control shRNA (KR42959N, SABiosciences, Frederick, Maryland, USA) were mixed with i-Fect ${ }^{\mathrm{TM}}$ (NI35150, Neuromics, Edina, Minnesota, USA) in a 1:5 ratio $(w / v)$ for intrathecal injection. Rats received $6 \mu \mathrm{g} / \mathrm{kg}$ in a 10- $\mu \mathrm{L}$ volume of either TRPV1 shRNA or control shRNA once daily for three consecutive days. The catheter was immediately flushed with $10 \mu \mathrm{L}$ of saline following every injection to ensure that all drugs reached the subarachnoid space.

Measurements of systolic blood pressure, water intake, and urine excretion

Systolic blood pressure of conscious rats was measured by the indirect tail-cuff method with a sphygmomanometer (Hatteras Instruments, Cary, North Carolina, USA). Rats were accustomed to the testing environment for $3 \mathrm{~d}$ prior to the measurement, and the blood pressure value for each rat was calculated as the average of nine separate measurements. Twenty-fourhour water intake and urine excretion were determined by the use of metabolic cages one day before and three days after intrathecal injection.

Measurement of mean arterial pressure (MAP) and its response to intravenous injection of capsaicin

The jugular vein and carotid artery of pentobarbital (50 $\mathrm{mg} / \mathrm{kg}$, ip) anesthetized rats were cannulated for administration of capsaicin $(30 \mu \mathrm{g} / \mathrm{kg}$, Sigma, M2028, dissolved in saline with $0.1 \%$ ethanol) and for monitoring of MAP (Gould Instruments, Valley View, Ohio, USA), respectively. MAP was recorded $30 \mathrm{~min}$ after the cannulation and recorded every $3 \mathrm{~s}$. The MAP value for each rat was calculated as the average of 10 min of continuous measurements.

\section{Blockade of $\alpha 1$-adrenoceptor by prazosin}

Prazosin (P7791, Sigma-Aldrich, St Louis, Missouri, USA, dissolved in saline), an a1-adrenoceptor blocker, was intragastrically administered via gavage, at a dose of $0.5 \mathrm{mg} / \mathrm{kg}$ of each administration dose, twice per day for $7 \mathrm{~d}$ starting $3 \mathrm{~d}$ before shRNA injection.

\section{Immunohistochemistry}

For TRPV1 staining, samples of the spinal cord and DRG of T8-L3 segments, mesenteric arteries, and kidneys were fixed in $4 \%$ paraformaldehyde dissolved in phosphate-buffered saline and then cut into $20-\mu \mathrm{m}$ sections using a cryostat (Leica CM 1850, Leica Biosystems Nussloch GmbH, Nussloch, Germany). The sections were incubated with guinea pig anti-TRPV1 polyclonal antibody (1:500, AB5566, Millipore, Billerica, Massachusetts, USA) for $1 \mathrm{~h}$ at room temperature, and then they were incubated with rhodamine red ${ }^{\mathrm{TM}}-\mathrm{X}$ donkey anti-guinea pig IgG (1:100, 711-295-152, Jackson ImmunoResearch Laboratories, West Grove, Pennsylvania, USA) for $1 \mathrm{~h}$ at room temperature. Pictures were taken with a fluorescent microscope (Olympus BX41 model, Olympus Optical Co, Ltd, Tokyo, Japan; Olympus MicroSuite ${ }^{\mathrm{TM}}$-Basic software, Olympus Soft 
Imaging Solutions GmbH, Münster, Germany).

For tyrosine hydroxylase staining, whole-mount mesenteric second-order arteries were fixed in $70 \%$ acetone (in saline) for $3 \mathrm{~h}$. The primary antibody was mouse anti-TH monoclonal antibody (1:1000, Calbiochem 657010, EMD Chemicals, Gibbstown, New Jersey, USA, 1:1000, $1 \mathrm{~h}$ at room temperature), and the secondary antibody was rhodamine red $^{\mathrm{TM}}-\mathrm{X}$ donkey anti-mouse IgG (1:100, 715-295-151, Jackson ImmunoResearch Laboratories, West Grove, Pennsylvania, USA, $1 \mathrm{~h}$ at room temperature).

\section{Western blot analysis}

Samples were homogenized in $10 \mathrm{mmol} / \mathrm{L}$ Tris buffer $(\mathrm{pH}$ 7.6) that included protein inhibitors. After centrifugation at $500 \times g$ for $5 \mathrm{~min}$, the supernatant was added to $20 \mu \mathrm{L}$ of 0.5 $\mathrm{mol} / \mathrm{L}$ EDTA and centrifuged at $50000 \times g$ for $1 \mathrm{~h}$. The pellet was resuspended with lysis buffer on ice for $45 \mathrm{~min}$ and centrifuged at $14000 \times g$ for $20 \mathrm{~min}$. After denaturation for $10 \mathrm{~min}$ at $100{ }^{\circ} \mathrm{C}, 30 \mu \mathrm{g}$ protein of each supernatant was electrophoresed on a 10\% SDS-PAGE gel and transferred to a PVDF membrane (162-0180, Bio-Rad Laboratories, Hercules, California, USA). Membranes were incubated with rabbit anti-TRPV1 (1:1000, PA1-29421, Affinity BioReagents, Golden, Colorado, USA) or mouse anti-TH (1:1000, 657010, Calbiochem, EMD Chemicals Inc, Gibbstown, New Jersey, USA) at $4{ }^{\circ} \mathrm{C}$ overnight, followed by incubation with HRP-donkey anti-rabbit IgG (1:10000, 711035-152, Jackson ImmunoResearch Laboratories, West Grove, Pennsylvania) or HRP-donkey anti-mouse IgG (1:10000, 711035-151, Jackson ImmunoResearch Laboratories, West Grove, Pennsylvania, USA) for $2 \mathrm{~h}$ at room temperature. The intensity of the bands was determined using ImageJ software (NIH, Bethesda, Maryland, USA). Protein loading on the membrane was normalized to $\beta$-actin (mouse anti- $\beta$-actin monoclonal antibody, 1:1000, sc-69879, Santa Cruz Biotechnology, Santa Cruz, California, USA).

\section{Statistical analysis}

Data are expressed as mean \pm SEM. Differences between paired groups were analyzed using an unpaired Student's $t$-test. Differences among groups were analyzed using a one-way ANOVA followed by a Bonferroni adjustment for multiple comparisons. Differences were considered statistically significant at $P<0.05$.

\section{Results}

Body weight in all groups increased with age. No difference in body weight was found between NS and HS diet-treated rats administered control or TRPV1 shRNA before dietary treatment, at the day of intrathecal injection, or $3 \mathrm{~d}$ after intrathecal injection (data not shown), which indicates that the general growth and condition of the rats were not affected by dietary or intrathecal treatment.

TRPV1 shRNA treatment resulted in a greater pressor effect in HS rats compared with NS rats

HS diet intake for 2 weeks, without shRNA treatment, increased systolic blood pressure when compared with NStreated rats (Figure 1A). Systolic blood pressure was elevated $3 \mathrm{~d}$ after intrathecal injection of TRPV1 shRNA in both NSand HS-treated rats (in NS rats, control shRNA: $126 \pm 2$ vs TRPV1 shRNA: $139 \pm 2 \mathrm{mmHg}, P<0.05$; in HS rats, control shRNA: $149 \pm 4$ vs TRPV1 shRNA: $169 \pm 3 \mathrm{mmHg}, P<0.05$, Figure 1A). Moreover, the increases in systolic blood pressure after TRPV1 shRNA treatment were greater in HS- than in NStreated rats (NS: $9.8 \% \pm 0.7 \%$ vs HS: $13.9 \% \pm 1.8 \%, P<0.05$, Figure 1B).
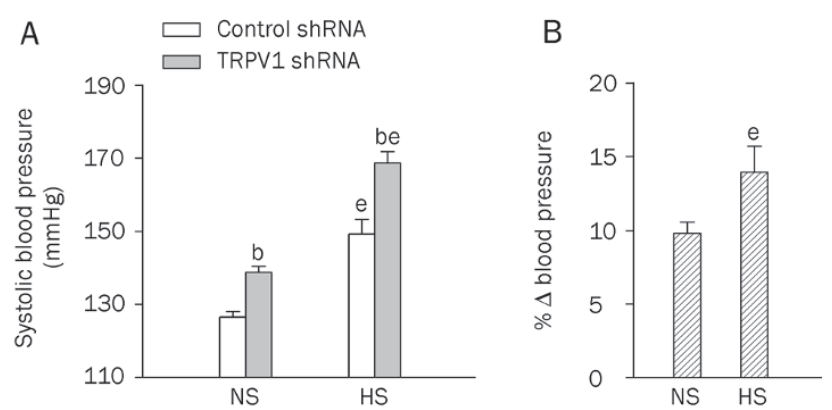

Figure 1. Effects of intrathecal injection of TRPV1 shRNA on systolic blood pressure in rats fed a normal-sodium (NS) or high-sodium (HS) diet. (A) The systolic blood pressure at d 3 after control or TRPV1 shRNA treatment. (B) The percentage change in systolic blood pressure of TRPV1 shRNA compared to that of corresponding control shRNA. Values are expressed as mean \pm SEM ( $n=11$ to 14$)$. ${ }^{b} P<0.05$ compared with the corresponding control shRNA-treated group; ${ }^{e} P<0.05$ compared with the corresponding NS diet group.

TRPV1 shRNA treatment led to greater TRPV1 downregulation in targeted tissues in HS rats compared with NS rats

Immunohistochemistry studies revealed that TRPV1 expression in the dorsal horn of the spinal cord (T8-L3 segments), DRG (T8-L3 segments), and mesenteric arteries was attenuated in TRPV1 shRNA-treated rats fed either an NS or a HS diet compared with corresponding control shRNA-treated rats (Figure 2). Consistently, quantitative measurement of TRPV1 content by Western blot analysis revealed that TRPV1 expression was knocked down by TRPV1 shRNA treatment in both NS rats and HS rats in the dorsal horn of the spinal cord (T8-L3 segments), DRG (T8-L3 segments), and mesenteric arteries (Figure 3). Western blot analysis revealed that TRPV1 expression was also knocked down by TRPV1 shRNA in the kidney (NS, control shRNA: $0.37 \pm 0.02$ vs TRPV1 shRNA: $0.29 \pm 0.01, P<0.05$; HS, control shRNA: $0.50 \pm 0.03$ vs TRPV1 shRNA: $0.29 \pm 0.02, P<0.05)$. In contrast, no significant change was found in TRPV1 levels in the cervical spinal cord in NS or HS rats after TRPV1 shRNA treatment (NS, control shRNA: $0.35 \pm 0.02$ vs TRPV1 shRNA: $0.32 \pm 0.03, P>0.05$; HS, control shRNA: $0.45 \pm 0.01$ vs TRPV1 shRNA: $0.44 \pm 0.02, P>0.05$ ), indicating that shRNA suppression occurred only in the targeted segments/tissues. Moreover, TRPV1 expression in the dorsal horn, DRG, and mesenteric arteries was higher in HS rats 


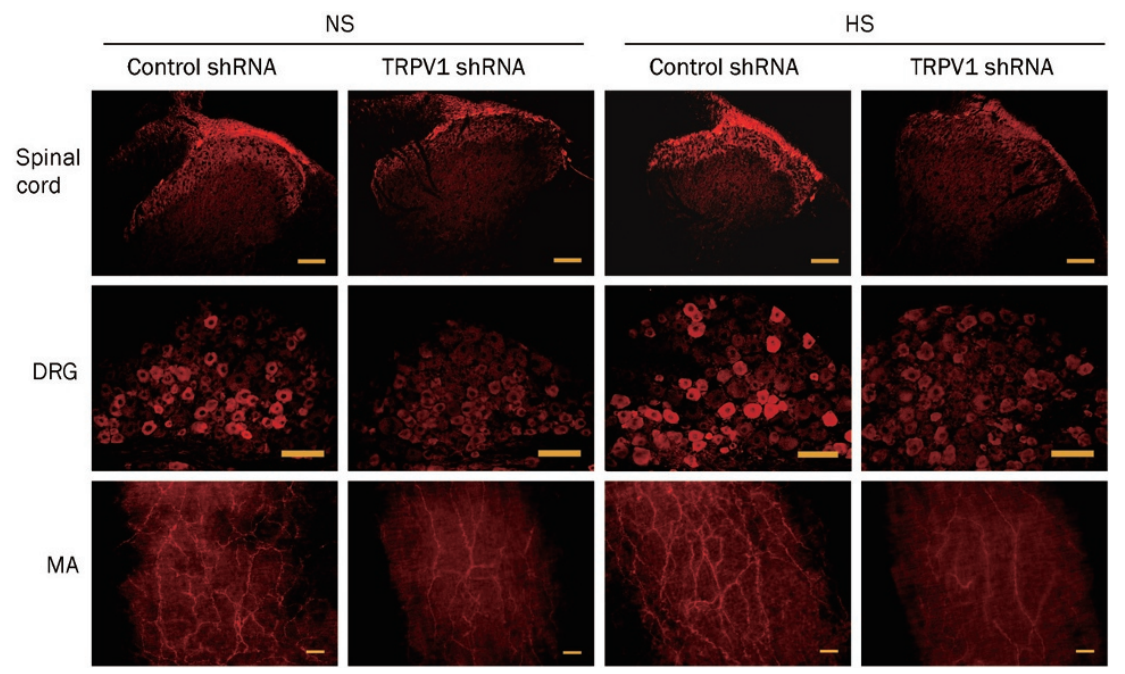

Figure 2. Immunohistochemistry staining showing TRPV1 expression in the dorsal horn of the spinal cord (T8-L3), dorsal root ganglia (DRG) (T8-L3) and mesenteric arteries (MA) of rats fed a NS or HS diet $3 \mathrm{~d}$ after control or TRPV1 shRNA treatment. Scale bars, $100 \mu \mathrm{m}$.

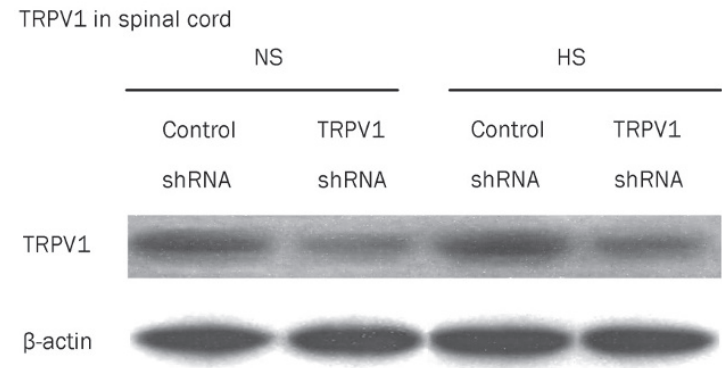

TRPV1 in DRG

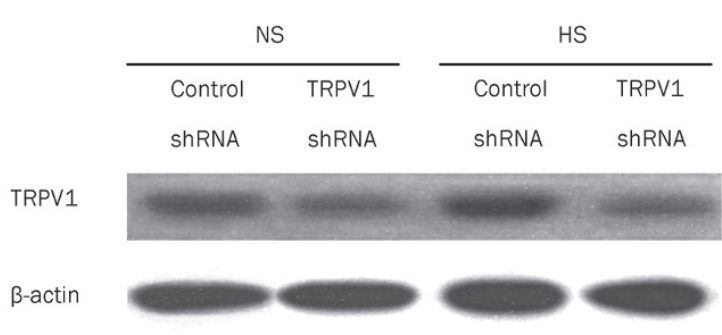

TRPV1 in mesenteric arteries

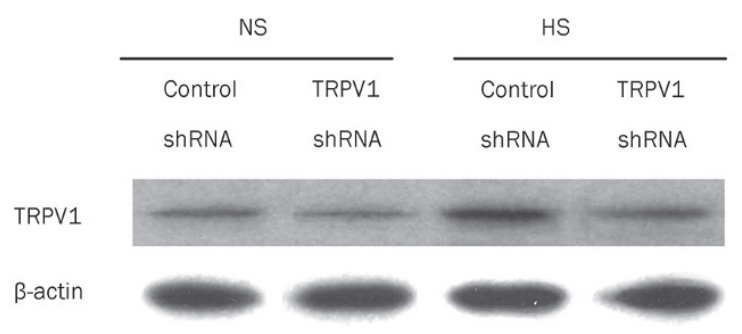

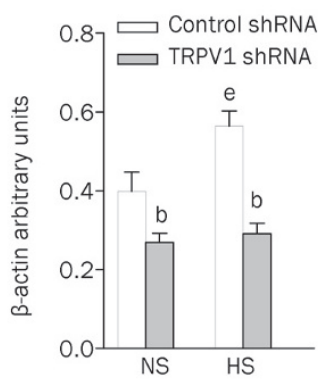
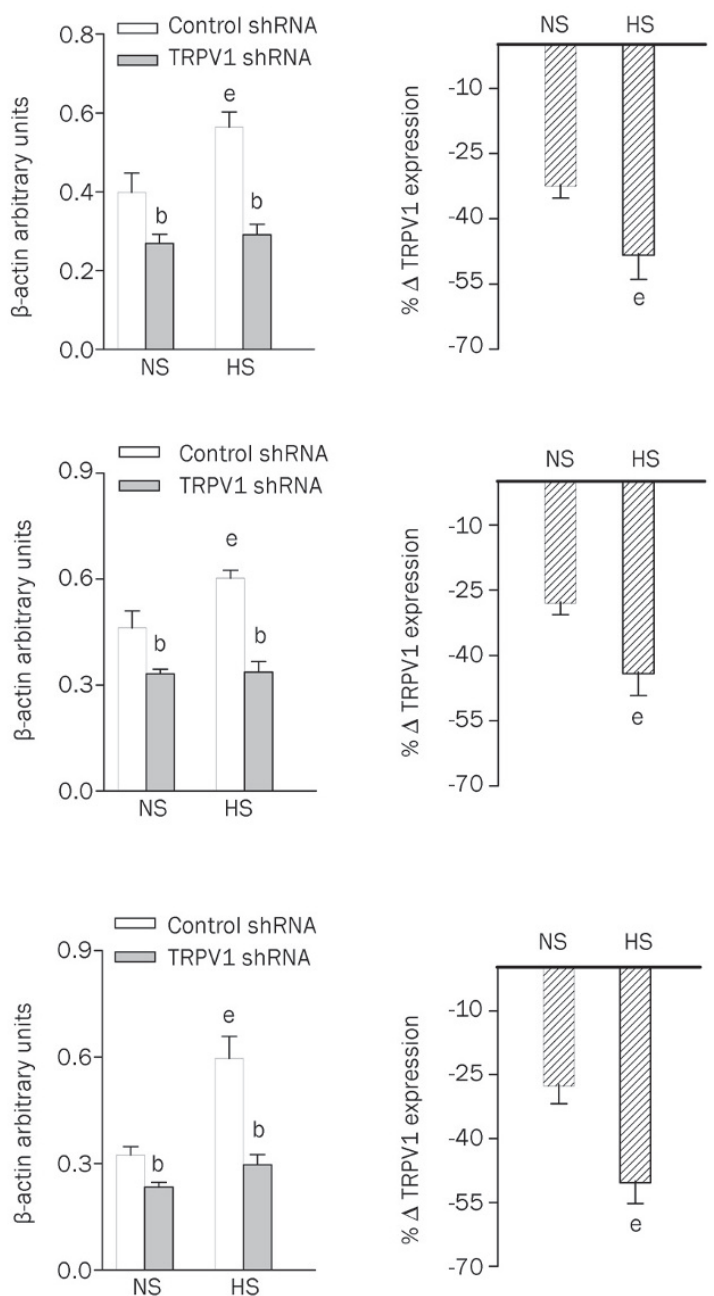

Figure 3. Western blot analysis showing TRPV1 expression in the dorsal horn of the spinal cord (T8-L3), DRG (T8-L3), and mesenteric arteries of rats fed a NS or HS diet $3 \mathrm{~d}$ after control or TRPV1 shRNA treatment. The left, middle, and right panels indicate representative Western blots, quantification results ( $\beta$-actin arbitrary units), and the percentage change of TRPV1 expression of TRPV1 shRNA from that of corresponding control shRNA, respectively. Values are expressed as mean \pm SEM $(n=5)$. ${ }^{b} P<0.05$ compared with the corresponding control shRNA-treated group; ${ }^{e} P<0.05$ compared with the corresponding NS diet group. 
administered control shRNA compared with NS rats given control shRNA, and the magnitude of TRPV1 down-regulation by TRPV1 shRNA treatment was greater in HS than in NS rats (dorsal horn, NS: $-32 \% \pm 3 \%$ vs HS: $-48 \% \pm 6 \%, P<0.05$; DRG, NS: $-28 \% \pm 3 \%$ vs HS: $-44 \% \pm 5 \%, P<0.05$; mesenteric arteries, NS: $-28 \% \pm 4 \%$ vs HS: $-50 \% \pm 5 \%, P<0.05)$.

TRPV1 shRNA treatment resulted in a greater degree of inhibition of capsaicin-induced depressor effects in HS rats compared with NS rats

To further confirm TRPV1 suppression induced by TRPV1 shRNA, we examined blood pressure responses to iv injection of capsaicin, a selective TRPV1 agonist. The magnitude of the prolonged depression phase of MAP in response to capsaicin was attenuated in both NS and HS rats (Figure 4A) that received TRPV1 shRNA compared with rats that received control shRNA. The magnitude of inhibition of capsaicin-induced depressor effects by TRPV1 shRNA treatment was greater in HS rats than in NS rats (NS: $-34 \% \pm 3 \%$ vs HS: $-50 \% \pm 3 \%, P<0.05$ ) (Figure 4B).
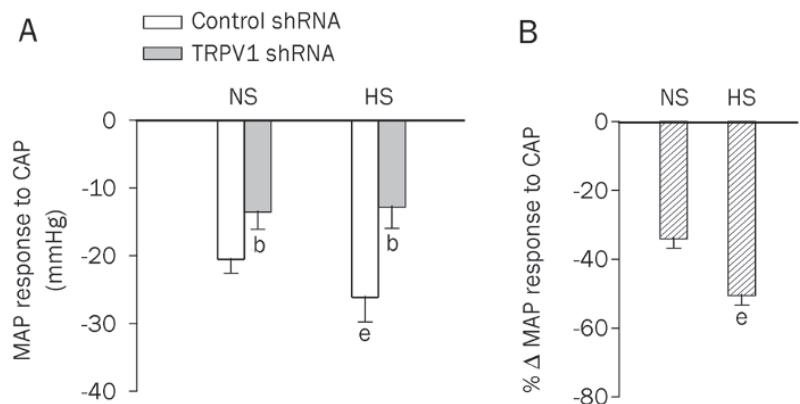

Figure 4. The response of mean arterial pressure (MAP) at $36 \mathrm{~s}$ to a bolus injection of capsaicin $(30 \mu \mathrm{g} / \mathrm{kg}$, iv) in NS rats and HS rats $3 \mathrm{~d}$ after administration of control or TRPV1 shRNA (A) and the percentage change of MAP in response to capsaicin following TRPV1 shRNA treatment compared with corresponding control shRNA (B). Values are expressed as mean \pm SEM ( $n=6$ to 7 ). ${ }^{b} P<0.05$ compared with the corresponding control shRNA-treated group; ${ }^{e} P<0.05$ compared with the corresponding NS diet group.

TRPV1 shRNA treatment had no effect on urinary excretion in HS- or NS-treated rats

HS intake increased urine excretion, water intake, and the ratio of urine/water intake compared with NS-treated rats both before shRNA treatment and $3 \mathrm{~d}$ after. However, no significant differences were found between control shRNA and TRPV1 shRNA-treated rats fed a NS or HS diet in 24-h urine excretion (NS rats, control shRNA: $30 \pm 1$ vs TRPV1 shRNA: $27 \pm 2 \mathrm{~mL}, P>0.05$; HS rats, control shRNA: $106 \pm 7$ vs TRPV1 shRNA: $106 \pm 9 \mathrm{~mL}, P>0.05$ ), 24 -h water intake (NS rats, control shRNA: $53 \pm 2$ vs TRPV1 shRNA: $50 \pm 4, P>0.05$; HS rats, control shRNA: $135 \pm 9$ vs TRPV1 shRNA: $131 \pm 10, P>0.05)$, or the ratio of 24-h urine/water intake (NS rats, control shRNA: $0.58 \pm 0.02$ vs TRPV1 shRNA: $0.54 \pm 0.02, P>0.05$; HS rats, control shRNA:
$0.79 \pm 0.01$ vs TRPV1 shRNA: $0.80 \pm 0.02, P>0.05)$.

Depressor effects induced by blockade of $\alpha 1$-adrenoceptors following treatment with TRPV1 shRNA were greater in HS rats compared with NS rats

In both NS and HS rats, blockade of the a1-adrenoceptor by prazosin pretreatment prevented TRPV1 shRNA-induced elevation of blood pressure (Figure 5A). Moreover, prazosin played a greater depressor role in HS-treated rats given TRPV1 shRNA compared with NS-treated rats given TRPV1 shRNA (NS: $-7 \% \pm 1 \%$ vs HS: $-12 \% \pm 1 \%, P<0.05$, Figure $5 \mathrm{~B}$ ).

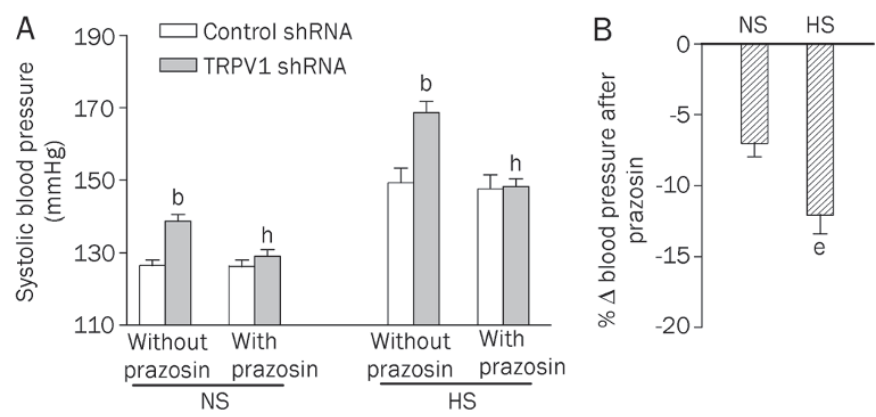

Figure 5. Pretreatment with prazosin prevented TRPV1 shRNAinduced elevation of blood pressure in rats fed NS or HS diets (A) and the percentage change of systolic blood pressure induced by prazosin compared with controls that did not receive prazosin (B). Values are expressed as mean \pm SEM ( $n=6$ to 7 ). ${ }^{b} P<0.05$ compared with the corresponding control shRNA-treated group; ${ }^{e} P<0.05$ compared with the corresponding NS diet group; ${ }^{h} P<0.05$ compared with the corresponding "without prazosin group."

TRPV1 shRNA treatment led to enhanced upregulation of mesenteric tyrosine hydroxylase in HS rats compared with NS rats

Immunohistochemistry staining (Figure 6A) and Western blot analysis (Figure 6B) revealed that TH levels in mesenteric arteries were enhanced in both NS and HS rats administered TRPV1 shRNA compared with corresponding control shRNA-treated rats. In control shRNA-treated rats, HS intake increased TH expression in mesenteric arteries compared with the NS diet. TRPV1 shRNA treatment produced a greater increase in TH in HS rats than in NS rats (NS: $46 \% \pm 9 \%$ vs HS: $77 \% \pm 6 \%, P<0.05$ ) (Figure $6 \mathrm{~B}$ ). In contrast, no significant differences were found in TH levels in the kidney in NS or HS rats administered TRPV1 shRNA compared with rats receiving the control shRNA (NS, control shRNA: $0.22 \pm 0.01$ vs TRPV1 shRNA: $0.24 \pm 0.01, P>0.05$; HS, control shRNA: $0.25 \pm 0.02$ vs TRPV1 shRNA: $0.30 \pm 0.02, P>0.05)$.

\section{Discussion}

The goal of this study was to determine whether selective knockdown of TRPV1 expressed in the DRG of lower thoracic and upper lumbar segments (T8-L3) and their central and periphery projections enhances prohypertensive effects induced by salt loading. We observed that (1) TRPV1 shRNA 
A
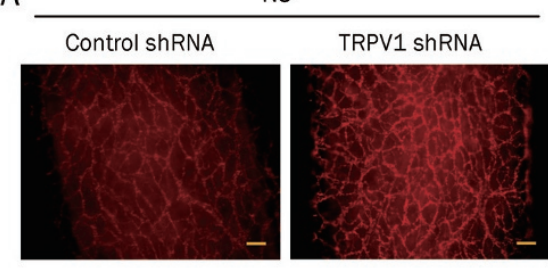

B

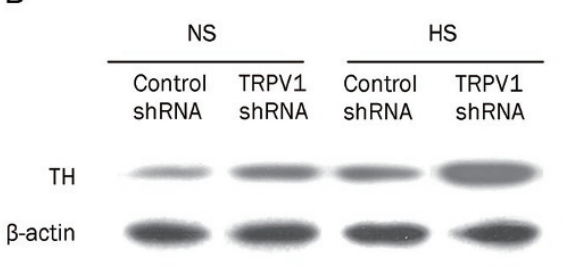

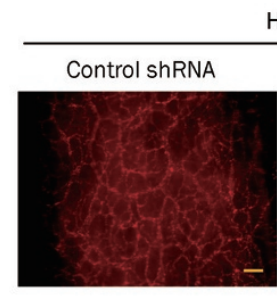

$\square$ Control shRNA

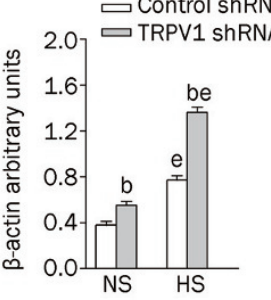

HS
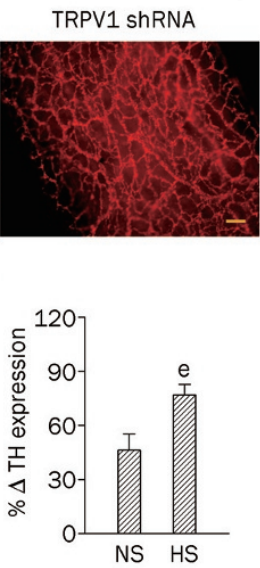

Figure 6. Immunohistochemistry staining (A) and Western blot analysis (B) showing tyrosine hydroxylase expression in mesenteric arteries of rats fed a NS or HS diet $3 \mathrm{~d}$ after either control or TRPV1 shRNA treatment. The left, middle, and right panels in $(B)$ indicate representative Western blots, quantification results (\% $\beta$-actin arbitrary units), and the percentage change of TH expression in rats receiving TRPV1 shRNA compared with control shRNA, respectively. Values are expressed as mean \pm SEM $(n=5)$. ${ }^{\mathrm{b}} \mathrm{P}<0.05$ compared with the corresponding control shRNA-treated group; ${ }^{e} P<0.05$ compared with the corresponding NS diet group. Scale bars, $100 \mu \mathrm{m}$. treatment increased blood pressure to a greater extent in HS rats compared with NS rats; (2) HS diet enhanced TRPV1 expression in the dorsal horn, DRG, mesenteric arteries, and kidneys, which was suppressed by TRPV1 shRNA treatment; (3) TRPV1 shRNA treatment attenuated the depressor action of capsaicin with a greater effect in HS rats compared with NS rats; (4) TRPV1 shRNA treatment had no effect on urinary excretion, water intake, or the ratio of urine/water intake in rats fed a NS or HS diet; (5) blockade of a1-adrenoceptors led to a greater depressor effect in HS rats compared with NS rats treated with TRPV1 shRNA; and (6) TRPV1 shRNA treatment produced a greater increase in $\mathrm{TH}$ in mesenteric arteries, but not kidneys, in HS rats compared with NS rats. These data show that selective knockdown of neuronal TRPV1 enhances prohypertensive effects induced by salt loading and that pressor effects of TRPV1 shRNA may be mediated, at least in part, by enhancement of the sympatho-excitatory response.

We have previously shown that neonatal degeneration of TRPV1-expressing sensory nerves increased salt sensitivity of arterial pressure as these rats grew into adulthood ${ }^{[6-20]}$. However, the systemic sensory denervation used in these previous studies precluded us from making conclusions about whether the observed effect was due to the removal of TRPV1 or other proteins co-expressed in the same nerves innervating any specific organs or tissues ${ }^{[21,22]}$. In the present study, we anatomically limited the TRPV1 affected by intrathecally injecting TRPV1 shRNA to selectively knockdown TRPV1 in an attempt to answer the question of whether impairment of TRPV1 in selective tissues is sufficient to enhance salt sensitivity of blood pressure. shRNA knocks down the expression of a specific gene by RNA interference, and its transfection efficacy can be further enhanced by the use of the transfection reagent $\mathrm{iFect}^{\mathrm{TM}}$, which is particularly designed for facilitating effective transfection for neuronal cells. We did not observe any neuron damage or other side effects following intrathecal injection of shRNA (data not shown), which is consistent with observations made by other laboratories ${ }^{[30]}$. Our results show that knocking down TRPV1 expression in T8-L3 DRG and their central and periphery projections, without affecting other segments such as the cervical spinal cord, significantly modulated the development of hypertension, especially during salt loading. These results indicate that intrathecal injection with shRNA is a viable strategy of conditional knockdown, which can be used to study the role of a given gene or protein.

Although TRPV1 expression in the kidney was suppressed, no changes occurred for 24-h urine excretion, water intake, or the ratio of urine/water intake after TRPV1 shRNA treatment. Although unexpected, these results suggest that mechanisms other than altered renal function are responsible for the prohypertensive effect of TRPV1 shRNA. Given that the peripheral projections of T8-L3 DRGs innervate mesenteric arteries, we examined mesenteric TRPV1 levels. Indeed, TRPV1 levels in mesenteric resistant arteries were suppressed by TRPV1 shRNA treatment. Interestingly, levels of tyrosine hydroxylase, a sympathetic nerve marker, were markedly upregulated after TRPV1 shRNA treatment in mesenteric resistant arteries but not kidneys. Furthermore, blockade of a1-adrenoceptors completely abolished TRPV1 shRNA-induced prohypertensive effects. These data suggest that heightened sympathetic nerve activity occurred, which contributed to TRPV1 shRNAinduced hypertension. The pressor effect of TRPV1 shRNA may be mediated by enhanced vasoconstriction of mesenteric resistant arteries via at least two potential mechanisms. First, knockdown of TRPV1 in the dorsal horn would decrease signal input from periphery organs to the central nervous system via afferent primary neurons and, as a result, diminish the inhibitory effect of afferent nerves on sympathetic nerve activity in mesenteric resistant arteries ${ }^{[31-34]}$. Second, knockdown of TRPV1 expressed in mesenteric resistant arteries would reduce the release of vasodilator neuropeptides such as CGRP and, as a result, diminish the counter-regulatory role of afferent nerves against vasoconstriction ${ }^{[24,35,36]}$.

Our data show that HS intake led to compensatory increases in mesenteric TRPV1 levels, which is consistent with previous reports $^{[36-38]}$. Moreover, the depressor effect of TRPV1 activation after capsaicin administration was much greater in HS rats compared with NS rats, supporting the notion that TRPV1 plays a counter-regulatory role against salt-induced increases 
in blood pressure. The counter-regulatory effect of TRPV1 has been shown to involve enhanced CGRP release from sensory nerves that cause vasodilation, sodium and water excretion, and decreases in blood pressure ${ }^{[39-43]}$. TRPV1 shRNA treatment resulted in enhanced suppression of mesenteric TRPV1 levels but enhanced up-regulation of mesenteric tyrosine hydroxylase contents. The combination of these effects would lead to a more pronounced pressor action in HS rats. Indeed, as shown in the present study, HS rats displayed a greater degree of pressor effects after TRPV1 shRNA treatment when compared with NS rats receiving the same treatment.

In conclusion, the present study shows that intrathecal injection of TRPV1 shRNA selectively knocks down TRPV1 expression in the DRG of T8-L3 segments and their central and periphery projections in rats, which enhances the prohypertensive effects induced by salt loading. The pressor effect of TRPV1 shRNA may be mediated, at least in part, by enhancement of the sympatho-excitatory response.

\section{Acknowledgements}

This work was supported in part by the NIH (grants HL-57853, HL-73287, and DK67620).

\section{Author contribution}

Donna H WANG designed research, secured funding, and interpreted the data; Shuang-quan YU performed the experiments and analyzed the data; Donna H WANG and Shuangquan YU wrote the paper.

\section{References}

1 Zygmunt PM, Petersson J, Andersson DA, Chuang H, Sorgard M, Di Marzo V, et al. Vanilloid receptors on sensory nerves mediate the vasodilator action of anandamide. Nature 1999; 400: 452-7.

2 Zahner MR, Li DP, Chen SR, Pan HL. Cardiac vanilloid receptor 1-expressing afferent nerves and their role in the cardiogenic sympathetic reflex in rats. J Physiol 2003; 551: 515-23.

3 Kopp UC, Jones SY, DiBona GF. Afferent renal denervation impairs baroreflex control of efferent renal sympathetic nerve activity. Am J Physiol Regul Integr Comp Physiol 2008; 295: R1882-90.

4 Caterina MJ, Schumacher MA, Tominaga M, Rosen TA, Levine JD, Julius D. The capsaicin receptor: a heat-activated ion channel in the pain pathway. Nature 1997; 389: 816-24.

5 Tominaga M, Caterina MJ, Malmberg AB, Rosen TA, Gilbert H, Skinner $\mathrm{K}$, et al. The cloned capsaicin receptor integrates multiple painproducing stimuli. Neuron 1998; 21: 531-43.

6 Wang DH, Li J, Qiu J. Salt-sensitive hypertension induced by sensory denervation: introduction of a new model. Hypertension 1998; 32: 649-53

7 Wang $\mathrm{DH}$, Li J. Antihypertensive mechanisms underlying a novel salt-sensitive hypertensive model induced by sensory denervation. Hypertension 1999; 33: 499-503.

8 Huang Y, Wang DH. Role of AT1 and AT2 receptor subtypes in saltsensitive hypertension induced by sensory nerve degeneration. J Hypertens 2001; 19: 1841-6.

9 Huang $\mathrm{Y}$, Wang DH. Role of renin-angiotensin-aldosterone system in salt-sensitive hypertension induced by sensory denervation. Am J Physiol Heart Circ Physiol 2001; 281: H2143-9.

10 Wang DH, Wu W, Lookingland KJ. Degeneration of capsaicin- sensitive sensory nerves leads to increased salt sensitivity through enhancement of sympathoexcitatory response. Hypertension 2001; 37: 440-3.

11 Ye DZ, Wang DH. Function and regulation of endothelin-1 and its receptors in salt sensitive hypertension induced by sensory nerve degeneration. Hypertension 2002; 39: 673-8.

12 Wang Y, Chen AF, Wang DH. ETA receptor blockade prevents renal dysfunction in salt-sensitive hypertension induced by sensory denervation. Am J Physiol Heart Circ Physiol 2005; 289: H2005-11.

13 Song WZ, Chen AF, Wang DH. Increased salt sensitivity induced by sensory denervation: role of superoxide. Acta Pharmacol Sin 2004; 25: 1626-32.

14 Wang Y, Chen AF, Wang DH. Enhanced oxidative stress in kidneys of salt-sensitive hypertension: role of sensory nerves. Am J Physiol Heart Circ Physiol 2006; 291: H3136-43.

$15 \mathrm{Li} \mathrm{J}$, Wang DH. Function and regulation of epithelial sodium transporters in the kidney of a salt-sensitive hypertensive rat model. J Hypertens 2007; 25: 1065-72.

16 Vaishnava P, Wang DH. Capsaicin sensitive-sensory nerves and blood pressure regulation. Curr Med Chem Cardiovasc Hematol Agents 2003; 1: 177-88.

17 Wang $\mathrm{DH}$. The vanilloid receptor and hypertension. Acta Pharmacol Sin 2005; 26: 286-94.

18 Wang Y, Wang DH. Neural control of blood pressure: focusing on capsaicin-sensitive sensory nerves. Cardiovasc Hematol Disord Drug Targets 2007; 7: 37-46.

19 Wang DH. Transient receptor potential vanilloid channels in hypertension, inflammation, and end organ damage: an imminent target of therapy for cardiovascular disease? Curr Opin Cardiol 2008; 23: 356-63.

20 Rayamajhi S, Contractor T, Wang DH. The potential of TRPV1 agonists for treating ischemia/reperfusion-induced renal injuries. Curr Opin Investig Drugs 2009; 10: 963-70.

21 Kobayashi K, Fukuoka T, Obata K, Yamanaka H, Dai Y, Tokunaga A, et al. Distinct expression of TRPM8, TRPA1, and TRPV1 mRNAs in rat primary afferent neurons with adelta/c-fibers and colocalization with trk receptors. J Comp Neurol 2005; 493: 596-606.

22 Price TJ, Flores CM. Critical evaluation of the colocalization between calcitonin gene-related peptide, substance $\mathrm{P}$, transient receptor potential vanilloid subfamily type 1 immunoreactivities, and isolectin B4 binding in primary afferent neurons of the rat and mouse. J Pain 2007; 8: 263-72.

23 Gao F, Sui D, Garavito RM, Worden RM, Wang DH. Salt intake augments hypotensive effects of transient receptor potential vanilloid 4: functional significance and implication. Hypertension 2009; 53: 228-35.

24 Kawasaki H, Takasaki K, Saito A, Goto K. Calcitonin gene-related peptide acts as a novel vasodilator neurotransmitter in mesenteric resistance vessels of the rat. Nature 1988; 335: 164-7.

25 Mayer G. An update on the relationship between the kidney, salt and hypertension. Wien Med Wochenschr 2008; 158: 365-9.

26 Ciriello J, Calaresu FR. Central projections of afferent renal fibers in the rat: an anterograde transport study of horseradish peroxidase. J Auton Nerv Syst 1983; 8: 273-85.

27 Donovan MK, Wyss JM, Winternitz SR. Localization of renal sensory neurons using the fluorescent dye technique. Brain Res 1983; 259: 119-22.

28 Chevendra V, Weaver LC. Distribution of splenic, mesenteric and renal neurons in sympathetic ganglia in rats. J Auton Nerv Syst 1991; 33: $47-53$

29 Huang J, Chowhdury SI, Weiss ML. Distribution of sympathetic 
preganglionic neurons innervating the kidney in the rat: PRV transneuronal tracing and serial reconstruction. Auton Neurosci 2002; 95: 57-70.

30 Zou W, Song Z, Guo Q, Liu C, Zhang Z, Zhang Y. Intrathecal lentiviralmediated RNA interference targeting PKCY attenuates chronic constriction injury-induced neuropathic pain in rats. Hum Gene Ther 2011; 22: 465-75.

31 Vizzard MA, Standish A, Ammons WS. Renal afferent input to ventrolateral medulla of the cat. Am J Physiol 1992; 263: R412-22.

32 Solano-Flores LP, Rosa-Arellano MP, Ciriello J. Fos induction in central structures after afferent renal stimulation. Brain Res 1997; 753: 102-19.

33 Bard P. Anatomical organization of the central nervous system in relation to control of the heart and blood vessels. Physiol Rev Suppl 1960; 4: 3-26.

34 Barman SM, Gebber GL. Brain stem neuronal types with activity patterns related to sympathetic nerve discharge. Am J Physiol 1981; 240: R335-47.

35 Wang LH, Luo M, Wang Y, Galligan JJ, Wang DH. Impaired vasodilation in response to perivascular nerve stimulation in mesenteric arteries of TRPV1-null mutant mice. J Hypertens 2006; 24: 2399-408.

36 Wang $\mathrm{Y}$, Wang $\mathrm{DH}$. Increased depressor response to $\mathrm{N}$-arachidonoyldopamine during high salt intake: role of the TRPV1 receptor. J
Hypertens 2007; 25: 2426-33.

$37 \mathrm{Li} \mathrm{J}$, Wang $\mathrm{DH}$. Function and regulation of the vanilloid receptor in rats fed a high salt diet. J Hypertens 2003; 21: 1525-30.

38 Wang Y, Kaminski NE, Wang DH. VR1-mediated depressor effects during high-salt intake: role of anandamide. Hypertension 2005; 46 : 986-91.

39 Wang Y, Novotny M, Quaiserová-Mocko V, Swain GM, Wang DH. TRPV1-mediated protection against endotoxin-induced hypotension and mortality in rats. Am J Physiol Regul Integr Comp Physiol 2008; 294: R1517-23.

40 Wang $\mathrm{Y}$, Wang $\mathrm{DH}$. A novel mechanism contributing to development of Dahl salt-sensitive hypertension: role of the transient receptor potential vanilloid type 1. Hypertension 2006; 47: 609-14.

41 Wang $\mathrm{DH}$, Zhao Y. Increased salt sensitivity induced by impairment of sensory nerves: is nephropathy the cause? J Hypertens 2003; 21 : 403-9.

$42 \mathrm{Li} \mathrm{J,} \mathrm{Wang} \mathrm{DH.} \mathrm{High-salt-induced} \mathrm{increase} \mathrm{in} \mathrm{blood} \mathrm{pressure:} \mathrm{role} \mathrm{of}$ capsaicin-sensitive sensory nerves. J Hypertens 2003; 21: 577-82.

43 Wang Y, Kaminski NE, Wang DH. Endocannabinoid regulates blood pressure via activation of the transient receptor potential vanilloid type 1 in Wistar rats fed a high-salt diet. J Pharmacol Exp Ther 2007; 321: 763-9. 